\section{Dos métodos para la adaptación transcultural de un instrumento psicométrico}

Para efectuar comparaciones entre estudios multicéntricos o internacionales, es cada vez mayor la necesidad de contar con versiones de un mismo instrumento psicométrico en distintos idiomas. En ocasiones se han efectuado traducciones a otros idiomas de instrumentos tales como la encuesta de salud SF36, las Tablas COOP, y el Perfil Sanitario de Nottingham, mientras que en otros casos los instrumentos se han elaborado en varios idiomas simultáneamente. Se ha visto que, por buena que sea una traducción, a menudo surgen problemas. Hasta el momento no se han efectuado estudios para investigar la idoneidad de las traducciones, o cuántas traducciones iniciales deben hacerse, quién debe efectuarlas, si las retrotraducciones tienen algún valor, o qué hacer cuando la traducción no refleja lo expresado en el original. De ahí que un grupo de investigadores se haya propuesto investigar las características de dos adaptaciones al francés de la encuesta de salud SF36. La primera, una traducción rápida elaborada en un período de 3 meses en 1992 por la Universidad de Ginebra, fue comparada con una traducción adaptada más extensa preparada en 1994 por el proyecto internacional para la Evaluación de la Calidad de Vida (International Quality of Life Assessment Project, IQOLA). Esta última se elaboró con la ayuda de retrotraducciones, grupos focales, desarrollo de respuestas equidistantes, pruebas preliminares y evaluaciones de la dificultad y calidad de las preguntas. A fin de determinar si con la traducción más extensa se obtenían mejores resultados psicométricos que con la más breve, ambos instrumentos se administraron en años consecutivos a un mismo grupo de 946 adultos.

Se encontraron diferencias en la redacción de 34 de los 36 ítems que integraban los instrumentos. Los efectos de "techo" fueron algo menores en el caso del IQOLA que en el de la versión suiza (medias: 30,4 y $35,5 \%$ ), como también lo fueron las puntuaciones ausentes (medias: 0,5 y 1,2\%). Los efectos de "suelo" (medias: IQOLA, 2,7\%; Ginebra, 2,4\%), las proporciones de personas que dieron la misma respuesta (IQOLA, 93,4\%; Ginebra, 94\%) y los coeficientes de consistencia interna (IQOLA, 0,78-0,89; Ginebra, 0,80-0,92) fueron similares. El análisis factorial corroboró la presencia en ambas traducciones de dos aspectos principales de la salud, el físico y el mental. En resumen, ambas versiones francesas de la encuesta de salud SF36 mostraron propiedades psicométricas similares, pese a las grandes diferencias en su elaboración. Esto implica que una traducción efectuada mediante un proceso de complejidad mediana puede dar resultados adecuados. Se necesitan estudios empíricos adicionales para poder determinar con qué métodos de traducción se obtienen los mejores resultados. (Perneger TV, Leplege, Etter J-F. Cross-cultural adaptation of a psychometric instrument: two methods compared. J Clin Epidemiol 1999;52(11):1037-1046.)

\section{La influencia de las malas experiencias en la infancia en el hábito de fumar}

El descenso acelerado del hábito de fumar que se venía observando en la población adulta estadounidense en los tres últimos decenios se ha detenido casi por completo, e incluso se empieza a observar un aumento paulatino del tabaquismo entre la juventud. Estas tendencias alarmantes se producen pese a los intentos por reducir el acceso a los cigarrillos y por contrarrestar los efectos de la propaganda, de la presencia en el hogar de parientes fumadores y de la presión de los amigos. Es posible que las tendencias observadas se deban parcialmente a las gigantescas campañas propagandísticas de las compañías tabacaleras, especialmente a la promoción de marcas que gustan a la juventud, y a una exposición cada vez más intensa al cigarrillo por los medios de comunicación. Pero otros factores, hasta ahora poco comprendidos y estudiados, podrían sumarse a estos. Se ha detectado una asociación entre el consumo prolongado de nicotina y la necesidad de automedicarse para sobrellevar el efecto de experiencias emocionales y sociales adversas durante la niñez. Deseosos de explorar más a fondo esta posible asociación, investigadores de los Centros para el Control y la Prevención de Enfermedades en Atlanta, Georgia, Estados Unidos de América, se basaron en un estudio retrospectivo de cohorte sobre las malas experiencias infantiles ( $A d$ verse Childhood Experiences [ACE] Study) para estimar la fuerza de la asociación entre dichas experiencias y cinco conductas relacionadas con el tabaquismo: 1) la iniciación a edad temprana; 2) la iniciación en la adultez; 3) el haber sido fumador en algún momento; 4) el ser fumador en el momento 
del estudio y 5) el ser gran fumador. Se tuvieron en cuenta ocho experiencias infantiles adversas, que son frecuentes en la sociedad: 1-3) el abuso sexual, físico o emocional; 4) el haber presenciado violencia física contra la madre; 5) el ser hijo de padres divorciados o separados; y 6-8) el haberse criado en un hogar con parientes encarcelados, mentalmente inestables, o adictos al alcohol $\mathrm{u}$ otras sustancias.

Según los resultados, uno o más de los ocho tipos de experiencias adversas en la infancia fueron notificados por $63 \%$ de los sujetos estudiados. Aun después de hacer ajustes por edad, sexo, raza y escolaridad, se observó un aumento del riesgo de fumar en cada una de las categorías. Al ser comparados con los sujetos que no notificaron malas experiencias durante la niñez, las personas que notificaron 5 o más de dichas experiencias tuvieron un incremento notable del riesgo de empezar a fumar a edad temprana (razón de posibilidades [RP]: 5,4; intervalo de confianza de 95\% [IC95\%]: 4,1 a 7,1); de haber sido fumador en algún momento (RP: 3,1; IC95\%: 2,6 a 3,8); de ser fumador en el momento del estudio (RP: 2,1; IC95\%: 1,6 a 2,7); y de ser gran fumador (RP: 2,8; IC95\%: 1,9 a 4,2). Cada asociación observada entre el hábito de fumar y el número de experiencias adversas durante la niñez fue intensa y directa $(P<0,001)$. Independientemente del número de experiencias adversas en la infancia, la presencia reciente de problemas acompañados de un componente depresivo se detectó con más frecuencia entre los fumadores que entre las personas que no fumaban.

Estos datos confirman que existe una estrecha asociación entre el tabaquismo y las malas experiencias en etapa temprana de la vida. Se infiere que las medidas de prevención primaria contra tales experiencias, así como el tratamiento adecuado de los niños expuestos, podrían reducir el hábito de fumar tanto en la adolescencia como en la adultez. (Anda RF, Croft JB, Felitti VJ, Nordenberg D, Giles WH, Williamson DF, et al. Adverse childhood experiences and smoking during adolescence and adulthood. JAMA 1999;282(17):1652-1658).

\section{Se puede predecir el riesgo de problemas cardiovasculares en ancianos hipertensos}

El uso de un monitor ambulatorio permite registrar la tensión arterial durante el día entero mientras los pacientes atienden a sus actividades cotidianas. También ofrece otras ventajas: se elimina el sesgo del observador, la tendencia a redondear las cifras, y el efecto del nerviosismo en presencia del médico u otro profesional de la salud. Para que la medición de la tensión arterial por el método tradicional arroje información de igual calidad, las lecturas deben ser estandarizadas y muy frecuentes. Por otra parte, varios estudios indican que la tensión arterial obtenida por monitoreo continuo muestra mayor correlación con la organopatía hipertensiva, como la hipertrofia ventricular izquierda, que la obtenida por el método tradicional.

Se llevó a cabo un estudio, basado en el Ensayo Europeo sobre Hipertensión Sistólica (Syst-Eur), en 808 personas de 60 años de edad o mayores con una tensión sistólica basal de 160 a 219 mmHg y una tensión diastólica basal de menos de $95 \mathrm{mmHg}$, ambas medidas por el método tradicional con el paciente sentado y en ausencia de tratamiento antihipertensivo. La tensión sistólica mínima con el paciente de pie debía ser de $140 \mathrm{mmHg}$. Estos criterios de inclusión se basaron en el promedio de seis mediciones (dos lecturas en cada visita, con un intervalo de un mes entre visitas). Los objetivos del estudio fueron comparar el valor pronóstico de la tensión arterial medida por el método tradicional y por monitoreo ambulatorio, así como calcular los umbrales con valor diagnóstico mediante el seguimiento de los pacientes para observar sus características de morbilidad y mortalidad en años posteriores. Los pacientes fueron asignados a doble ciego a uno de dos grupos: uno tratado con antihipertensivos (nitrendipina, sola o con elanapril, hidroclorotiazida, o ambos), y otro al que se administró placebo. La tensión arterial ambulatoria se midió al inicio del estudio y un año más tarde con monitores que tomaban mediciones con una frecuencia mínima de $30 \mathrm{minu}$ tos. Las variables de resultado fueron las siguientes: 1) la mortalidad total y por trastornos cardiovasculares; 2 ) la presencia de cualquier tipo de problema cardiovascular; 3) la presencia de accidente cerebrovascular mortal o no mortal; 4) y la presencia de trastornos cardíacos mortales o no mortales.

Tras hacer ajustes por sexo, edad, complicaciones cardiovasculares previas, tabaquismo y residencia en Europa occidental, se observó que en pacientes tratados con antihipertensivos un aumento diferencial de la tensión sistólica de $10 \mathrm{mmHg}$ en un período de 24 horas no se asoció con un peor pronóstico, mientras que en el grupo que recibió placebo el mismo aumento se asoció con un incremento significativo del riesgo relativo (RR) de presentar la mayor parte de las variables de resultado (RR: 1,23 para mortalidad total y 1,34 para mortalidad por causas cardiovasculares). En el grupo que recibió placebo, la tensión arterial sistólica nocturna tuvo mayor valor predictivo que la diurna. Fue mayor el riesgo de trastornos cardiovasculares en pacientes cuya razón entre la tensión sistólica nocturna y diurna era mayor, independientemente de su tensión arterial en 24 horas. El riesgo de sufrir trastornos cardiovasculares que se asoció con una tensión sistólica de $160 \mathrm{mmHg}$ determinada por el método tradicional fue similar a la asociada con una tensión 
sistólica obtenida en 24 horas, durante el día o durante la noche con el monitor ambulatorio de 142, 145 y $132 \mathrm{mmHg}$, respectivamente. En el grupo tratado con antihipertensivos, la tensión sistólica no mostró un valor predictivo significativo con respecto al riesgo de trastornos cardiovasculares, independientemente de la técnica usada para hacer la medición. En resumen, en pacientes de edad avanzada que solamente tienen hipertensión sistólica y que no reciben tratamiento con antihipertensivos, la tensión sistólica obtenida con monitor continuo tuvo un valor predictivo significativo de problemas cardiovasculares que no fue observado en el caso de la tensión sistólica obtenida por el método tradicional. (Staessen JA, Thijs L, Fagard R, O'Brien ET, Clement D, de Leeuw PW, et al. Predicting cardiovascular risk using conventional vs. ambulatory blood pressure in older patients with systolic hypertension. JAMA 1999;282(6):539-546).

\section{Tratamiento promisorio para las exacerbaciones agudas de la bronquitis crónica}

Las exacerbaciones agudas de la bronquitis crónica son frecuentes y ha habido cierta controversia sobre la utilidad de los antimicrobianos para su tratamiento. No obstante, algunos datos respaldan su uso en estos casos, siempre que se tengan presentes las siguientes características a la hora de elegir el antimicrobiano adecuado: buena actividad contra los patógenos bacterianos que se asocian con dichas exacerbaciones (Haemophilus influenzae no tipificable, Moraxella catarrhalis y los neumococos); resistencia a las betalactamasas bacterianas; fácil penetración en las secreciones y tejidos del árbol respiratorio; fácil administración; eficacia sin tendencia a provocar una respuesta inflamatoria intensa; y una elevada razón costo:eficacia. Las fluoroquinolonas reúnen estas características, pero la relativa ineficacia contra Streptococcus pneumoniae de algunas de las más antiguas cuando se administran a las dosis estándares limitan su uso para tratar las exacerbaciones agudas de la bronquitis.

Las fluoroquinolonas más nuevas, representadas por el levofloxacino, grepafloxacino, sparfloxacino y trovafloxacino, poseen gran actividad contra las bacterias Gram positivas y Gram negativas y las formas atípicas que suelen causar las exacerbaciones agudas de la bronquitis crónica. Se pueden administrar solo una vez al día, lo cual facilita el cumplimiento del paciente y aumenta las probabilidades de conseguir un buen resultado. Las reacciones adversas asociadas con cada fluoroquinolona nueva son raras y suelen limitarse a náusea y dispepsia, mareos, insomnio, fototoxicidad (en el caso de la sparfloxacina) y cefalea. (Niederman MS.
Contribución clínica de las nuevas fluoroquinolonas en las exacerbaciones agudas de la bronquitis crónica. Medicina (Buenos Aires) 1999;59(supl 1): 23-30.

\section{Resurgimiento de VIH al suspender el uso de IL-2 y antirretrovíricos combinados}

Según informaron recientemente algunos investigadores del Instituto Nacional de Alergias y Enfermedades Infecciosas (National Institute of Allergy and Infectious Diseases, NIAID), dos pacientes infectados por virus de la inmunodeficiencia humana (VIH) en tratamiento con combinaciones muy fuertes de medicamentos antirretrovíricos e interleukina-2 (IL-2) y en quienes las pruebas más sensibles no detectaban HIV viables volvieron a tener altas concentraciones víricas en la sangre a las tres semanas de haberse suspendido el tratamiento. Estos datos vienen a sumarse a informes anteriores de que no es posible erradicar los VIH del organismo con los tratamientos disponibles en la actualidad porque el virus puede alojarse en forma latente en recintos o "reservorios" donde no penetran los medicamentos y que se forman al poco tiempo de adquirirse la infección. Entre ellos se encuentran algunas células del sistema inmunitario, particularmente los linfocitos CD4+ inactivos del torrente sanguíneo y los ganglios linfáticos, y posiblemente algunas células del cerebro, el intestino, la médula ósea, los genitales y otros órganos. El virus se sigue replicando lentamente en estos reservorios, aun en presencia de un tratamiento intenso, y son los reservorios la fuente más probable de los VIH que reaparecen al suspenderse el tratamiento. El estudio del NIAID demostró que al menos el reservorio de células CD4+ inactivas con infección latente se vuelve a multiplicar rápidamente.

Estos datos apuntan a un hecho desalentador: la formación de reservorios víricos constituye un gran obstáculo al control a largo plazo de la infección por VIH y es preciso explorar regímenes terapéuticos más potentes o capaces de penetrar en dichos reservorios o de estimular la inmunidad específica contra VIH. (Chun TW, Davey RT, Engel D, Lane HC, Fauci AS. Re-emergence of HIV after stopping therapy. Nature 1999;401:874-875.)

\section{Los inhibidores de la recaptación de serotonina y la hemorragia gastrointestinal superior}

Se ha notificado en los últimos años una asociación entre los inhibidores selectivos de la recaptación de serotonina, como la fluoxetina, y los trastornos de la coagulación. La mayoría de los pacientes han te- 
nido hemorragias leves, pero también se han notificado casos de hemorragias de mayor gravedad en el aparato digestivo, las vías urinarias y el cerebro.

La liberación de serotonina por las plaquetas desempeña un papel importante en la regulación de la respuesta homeostática a las lesiones vasculares. La serotonina no se elabora en las plaquetas, pero estas poseen en la superficie moléculas especializadas que ligan y transportan la serotonina que circula en la sangre. A dosis terapéuticas, la fluoxetina y otros inhibidores selectivos de la recaptación de serotonina han mostrado la capacidad de bloquear esta actividad, lo cual conduce a un agotamiento de las reservas plaquetarias de serotonina al cabo de varias semanas de tratamiento. Por lo tanto es posible que estos medicamentos interfieran con la función hemostática, al menos en ciertas circunstancias, y que aumenten el riesgo de hemorragia.

La hipótesis fue puesta a prueba por investigadores españoles en un estudio de 1899 casos (1 651 con hemorragia gastrointestinal superior y 248 con úlcera perforada) y 10000 controles apareados por edad, sexo y año en que se identificó el caso. Los datos se obtuvieron por repaso de registros computadorizados de los casos tratados desde abril de 1993 hasta septiembre de 1997 en consultorios del Reino Unido, y se usó un estudio de validación interna para confirmar la exactitud del diagnóstico en la base de datos. Se usó como variable de resultado el haber consumido inhibidores selectivos de la recaptación de serotonina $\mathrm{u}$ otros antidepresivos en los 30 días anteriores a la fecha índice.

Los resultados revelaron una exposición a los inhibidores de la recaptación de serotonina en 3,1\% de los pacientes con hemorragia del aparato digestivo superior, a diferencia de $1,0 \%$ de los controles. No se observó ninguna influencia del sexo, la edad, la dosis o la duración del tratamiento. Se halló una asociación débil con los inhibidores no selectivos de la recaptación de serotonina, pero no con los antidepresivos que no tienen esta actividad inhibidora. Ninguna de las categorías de antidepresivos mostró asociación con la úlcera perforada, pero el uso simultáneo de inhibidores selectivos de la recaptación de serotonina y de antiinflamatorios no esteroideos aumentó el riesgo de hemorragia gastrointestinal superior más allá de la suma de sus efectos independientes (RR: 15,6; IC95\%: 6,6 a 36,6). También se halló una menor interacción entre los inhibidores selectivos de la recaptación de serotonina y la aspirina a dosis bajas (RR: 7,2; IC95\%: 3,1 a 17,1). Aunque estos datos indican la presencia de una asociación, el efecto absoluto es moderado y equivale al que se asocia con el ibuprofeno a dosis bajas. (de Abajo FJ, García Rodríguez LA, Montero D. Association between selective serotonin reuptake inhibitors and upper gastrointestinal bleeding: populationbased case-control study. BMJ 1999;319: 1106-1109). 